\title{
The Use of Fibrinolytic Therapy in Acute Ischemic Events
}

\author{
Danny Del Duca, B.Sc.*
}

\section{THE BASIS OF ISCHEMIC EVENTS}

The loss of tissue perfusion represents the pathological basis underlying acute cerebrovascular events. The occlusion of the brain's blood supply by intravascular thrombotic and/or atherosclerotic phenomena results in a disruption in tissue oxygen and glucose delivery. This inevitably impairs the cell's capacity to synthesize ATP. Despite initial attempts to maintain consistent ATP stores through anaerobic glycolysis and adenosine release, the starving tissue experiences loss of membrane ionic gradients, secondary to the inactivation of the $\mathrm{Na}+\mathrm{K}+$ ATPase pump. A significant increase in intracellular calcium concentration is triggered by the activation of voltagegated calcium channels, the activation of ligand-gated calcium channels, and by the inactivation of the $\mathrm{Na}+\mathrm{Ca}+$ exchanger. In addition, massive release of the main excitatory, inhibitory, and monoamine neurotransmitters (many of which become excitotoxins at high concentration) is also observed. The above excitatory transmitters, such as glutamine, for example, play an important role in the over-stimulation of the NMDA receptor, which in turn promotes excessive calcium influx (1). The cytotoxic effects of calcium are associated with the activation of lipases, proteases, and endonucleases, as well as xanthine oxidase and neuronal NO synthase, capable of either direct or indirect destruction of cellular structures (2).

\footnotetext{
*To whom correspondence should be addressed: Faculty of Medicine, McGill University, Montreal, Quebec, Canada H3G 1Y6; Email: Deldu04@med.mcgill.ca.
}

\section{RECOMBINANT TISSUE PLASMINOGEN ACTIVATOR}

The therapeutic basis for the use of recombinant tissue plasminogen activator (t-PA) rests in its capacity to interact with and enhance the function of plasmin, a nonspecific protease which digests fibrin. Plasmin is the central enzyme of the physiologic fibrinolytic system, and is formed from its inactive precursor, plasminogen, through the cleavage of a single peptide bond by various plasminogen activators including endothelial-derived $t-$ PA, urothelium-derived urokinase, streptokinase, and factor XIIa. Endogenous t-PA, a 527 amino acid serine protease, is released in response to stasis produced by vascular occlusion, and is largely specific for activating fibrin?bound plasminogen since it specifically associates with fibrin through the recognition of particular lysine residues (3). The site-sensitive nature of this agent has been viewed as a favorable aspect for therapeutic potential. However, the concentrations of $t$ PA achieved during pharmacotherapy far exceed those in the physiologic range, thereby reducing the likelihood that systemic activation of plasminogen will not occur. The action of t-PA is antagonized by plasminogen activator inhibitors- 1 and -2 . The half-life of the active species is 5 to 10 minutes, and clearance occurs chiefly by hepatic metabolism. Moreover, therapy with t-PA is expensive, costing several times more than streptokinase per therapeutic dose.

Despite the important potential for re-establishing blood flow to infarcted regions, one of the main limitations of the use of t-PA has been its association with hemorrhagic events, related to reperfusion injury, in certain patients exposed to this treatment. While it is 
clear that cell death occurs within the context of ischemic insult, variable numbers of cells may proceed to die after blood flow resumes, by necrosis as well as by apoptosis. It is hypothesized that cellular damage may be initiated during reoxygenation through the generation of oxygen free radicals by parenchymal and endothelial cells and infiltrating leukocytes. Cellular antioxidant defence mechanisms may also be compromised by ischemia, favoring the accumulation of free redicals. Finally, ischemic injury is associated with the production of cytokines and increased expression of adhesion molecules by hypoxic parenchymal and endothelial cells. These agents recruit circulating polymorphonuclear leukocytes to reperfused tissue; the ensuing inflammation causes additional injury (4). It follows that the above deterioration in the integrity of the reperfused tissue may result in hemorrhagic complications associated with thrombolytic therapy. The most likely mechanism is related to cerebral vascular dysfunction, and this represents the most significant limitation in the clinical use of t-PA in acute ischemic stroke. Other mechanisms which have been proposed to explain the mechanism of t?PA's hemorrhagic toxicity include the lysis of fibrin in physiological thrombi at sites of vascular injury, and the evolution of a systemic lytic state that results from the systemic activation of plasmin, which produces fibrinolysis and destruction of other coagulation factors, especially factors V and VIII (3). For this reason, the combination of neuroprotective pharmacotherapy with thrombolysis has been suggested as a means of reducing the untoward effects of reperfusion. Proposed methods include the concurrent management of t-PA patients with antiexcitotoxic, antiapoptotic, and/or free radical scavenging agents (5).

\section{RECENT FINDINGS AND ONGOING CLINICAL DEBATES}

The drug alteplase (tissue plasminogen activator) was recently approved for use in acute stroke in the United States (1996) and Canada (1999). According to national treatment guidelines, administration is recommended within three hours after the onset of ischemia, with the objective being the degradation of the intraarterial occlusion to restore blood perfusion before significant neuronal death ensues. However, the safety and efficacy of this therapy are still under scrutiny. In a recent survey of American neurologists, 30\% reported being concerned about its efficacy, while $61 \%$ admitted being concerned about the risk of intracranial hemorrhage, the most important adverse effect of t-PA regimens (6). Indeed, cerebral angiography conducted soon after the onset of most ischemic conditions has demonstrated the presence of arterial occlusion in up to $80 \%$ of acute infarctions (7). It follows, then, that attempts to promote clot degradation are well justified, given that such intervention can potentially limit brain injury if administered in a timely manner, and before the process of infarction has been completed.

\section{NINDS STUDY}

A large, randomized, placebo-controlled study (8) conducted by the National Institute of Neurological Disorders and Stroke (NINDS) in 1995 represented a landmark contribution in favor of this therapeutic approach, and played no small role in encouraging the nation-wide approval of alteplase in the US, shortly thereafter. The study, involving 624 patients treated with intravenous tissue plasminogen activator at $0.9 \mathrm{mg} / \mathrm{kg}$ body mass, within 3 hours of symptom onset, was divided into two parts. Part 1 measured changes in neurologic deficit 24 hours after the onset of stroke, as an indication of t-PA's immediate clinical effect, while Part 2 assessed different aspects of recovery at three months after treatment. Although no significant clinical improvement was observed within the first 24 hours, a statistically significant improvement in the condition of the t-PA-treated patients, compared to controls, was noted at three months. Patients treated with t-PA were $30 \%$ more likely to have minimal or no disability after three months of initial presentation. The beneficial effects occurred in patients with all subtypes of stroke, including suspected lacunar infarction, and were sustained at 1 year. This was accompanied by the finding that exposing patients to this therapy caused no significant differences in mortality ( $17 \%$ total mortality of treated patients versus $21 \%$ control). However, symptomatic intracerebral hemorrhage was seen to be associated with t-PA exposure. Within 36 hours after onset of stroke, hemorrhage took place in $6.4 \%$ of individuals given t-PA, but only in $0.6 \%$ of placebo cases, and at three mouths, $61 \%$ of those having hemorrhages died. The final results suggested that this therapy involved a $3 \%$ chance of early mortality attributable to intracerebral hemorrhage.

The NINDS study was among the first to indicate that earlier initiation of treatment and the use of lower doses might be important factors in reducing the incidence of t-PA-induced hemorrhagic events. Moreover, the sustained favorable clinical outcome after t-PA treatment was recently shown to be present in patients at 6 and 12 month periods after treatment (9), indicating a sustained benefit of t-PA for cases managed within three hours of initial insult. The importance of the administration of treatment within three hours of stroke onset was suggested by Clark et al. (10), whose work demonstrated that a similar treatment protocol followed at times beyond 3 hours 
of initial symptomology failed to show a benefit in individuals treated with t-PA compared to controls. The apparently favorable results of the NINDS work led to the development of a treatment protocol for t-PA which included the use of doses of $0.9 \mathrm{mg} / \mathrm{kg}$, administered no later than 3 hours after the onset of symptoms. In addition, the exclusion of patients deemed ineligible for this therapy was also stipulated. This involved the screening of treatment candidates for the presence of risk factors predisposing to the development of hemorrhagic complications with t?PA therapy, such as evidence of elevated blood pressure, advanced age, high blood glucose levels, or evidence of prior cerebral events on initial CT imaging studies. Indeed, as discussed below, there is evidence suggesting more favorable clinical outcomes in patients treated in concordance with this protocol, while most morbidity associated with t-PA may be caused by deviance from it.

\section{CLEVELAND STUDY}

This work (11) underlines the significant morbidity that has since been associated with t-PA, and has played no small role in creating skepticism among practitioners. However, it has also highlighted the importance of adequate patient selection in minimizing the incidence of hemorrhagic events. The study involved 3948 subjects from 29 Cleveland area hospitals, admitted over a one-year period (July 1997 to June 1998). Of these patients, 70 were treated with t-PA within three hours of initial insult, but $15.7 \%$ of these patients subsequently suffered symptomatic intracerebral hemorrhage. Despite the fact that the number of deaths associated with this latter complication was only $37 \%$ (generally lower than the above work), the overall higher rate of hemorrhagic complications is disturbing. In addition, the authors note that the in-hospital mortality rate for the t-PAtreated patients $(15.7 \%)$ was significantly higher than that for control patients not receiving the drug $(7.2 \%)$.

Katzan et al. (2000) state, most importantly, the fact that up to $50 \%$ of the t-PA patients in the above trial were reported to have been treated in a manner that deviated, in one or more ways, from the National Treatment Guidelines established following the NINDS (1995) work. This inclusion of high risk patients in the study is, indeed, thought to have played a significant role in the higher incidence of complication-related mortality, and only provides further proof of the importance of carefully screening patients before treatment is commenced. In fact, this finding is congruent with work by Karbalai et al. (12) who report on a series of 69 patients treated with intravenous t-PA. It was found that while the incidence of hemorrhage was as high as $27 \%$ in cases deviating from established protocol, the corresponding value was only 5\% for patients treated without deviations. Similarly, Tanne et al. (13) report that the incidence of hemorrhage was $11 \%$ in patients with protocol deviations, compared to $4 \%$ when no deviations were present, thereby suggesting that strict adherence to protocol guidelines is important.

\section{ECASS STUDIES}

Further insight unto the controversy surrounding t-PA may be gained be analyzing the results of the European Cooperative Acute Stroke Studies, ECASS I (14) and II (15). The ECASS I trial reported a $20 \%$ incidence of hemorrhagic events in the t-PA group, compared to $6.5 \%$ controls. Interestingly, this study differed significantly from the NINDS trial in several significant ways. Not only was thrombolytic therapy initiated up to 6 hours after the initial event, but the dose of alteplase was $1.1 \mathrm{mg} / \mathrm{kg}$ (compared to 0.9 ). In addition, $17 \%$ of patients included were in violation of the established treatment protocol. Furthermore, while no significant difference in primary end points was initially detected, if the above data are re-evaluated without those patients who should initially have been eliminated from the study, three-month disability scores are found to be significantly lower in the t-PA group (41\% vs. $29 \%)$. In 1998, ECASS II found no significant difference between patients receiving t-PA compared to controls, in terms of favorable outcome at three months. This may be related to the fact that the t-PA dose used was tapered to $0.9 \mathrm{mg} / \mathrm{kg}$, and that protocol violations were minimized through more effective identification of early infarct signs on pretreatment CT scan analysis, and blood pressure control. The study did, however, draw important conclusions related to additional patient risk factors for the development of hemorrhagic events. These included hypertension, prior congestive heart failure, advanced age, aspirin use prior to stroke, and central nervous system abnormalities (attenuation of density; edema; mass effect) on pretreatment CT scanning. Other studies have added elevated blood glucose levels (16) and evidence of a large infarct in the middle cerebral artery on CT scan (15) to this list.

Taken together, these data point to the unifying theme related to the importance of identifying and excluding high-risk patients during the initial stages of fibrinolytic therapy. Indeed, as efforts to find solutions to this seemingly subjective designation of high-risk patients, many authors have since advocated the development of predictive scoring algorithms that could standardize and perhaps facilitate clinical decision-making procedures. However, it is tempting to argue, as the results of the ECASS II trial demonstrate, that when all efforts to 
adhere to treatment protocol are followed, the ultimate statistical benefits of t-PA regimens become questionable. The current debate surrounding the use of t-PA reflects this notion.

\section{THE PROBLEM OF TIME}

Among the most important limitations associated with the use of t-PA remains the need to administer the treatment within the initial 3 hours following the acute insult, given the reported reduction in its efficacy as time-to-therapy in prolonged. In 1999, an important randomized controlled trial (17) failed to show any benefits of t-PA administered beyond the established three-hour window. Treatments at times ranging from 3 to 5 hours were associated with increased risk of symptomatic intracerebral hemorrhage, and no remarkable clinical benefits at 90 days. It follows that the advanced age of many of the patients affected often complicates the likelihood of prompt intervention, for reasons related to social isolation, and communication difficulties with care-providers. Indeed, it has been reported that, in one cohort of stroke patients, $29 \%$ of those presenting after three hours had recognized their symptoms but had chosen not to seek medical attention (18). Furthermore, efforts to enhance the specific diagnostic efficiency of acute care personnel in the area of acute cerebrovascular insult may also contribute to the more timely initiation of therapy.

\section{CONCLUSION}

One may thus infer that ethical implications can arise in the treatment of patients presenting with one or more of the above risk factors which would question their clinical eligibility for fibrinolytic therapy. Proponents of t-PA subscribe to the idea that treatment should not be withheld from patients if it can potentially result in a lower rate of severe disability, despite the fact that very favorable outcomes may be unlikely. Nevertheless, the enhanced probability of hemorrhage in patients presenting with the above risk factors clearly constitutes a challenge in the eyes of most clinical practitioners, and many currently favor a more conservative approach. Indeed, Hacke et al. (19) concluded that the use of widespread intravenous thrombolysis could not be advocated, based on the fact that treating high-risk patients is associated with an unacceptable tenfold increase in the risk of hemorrhagic complications and death.

It is thus safe to conclude that the proven benefits of thrombolytic therapy are reproducible in the clinical setting if guidelines for treatment and management, including the withholding of treatment in higher risk patients, are closely followed. However, while t-PA constitutes an important therapeutic option that is appropriate for some occlusive lesions, it is not indicated for all patients presenting with acute ischemic stroke. The problem of cerebrovascular disease has long posed an insurmountable obstacle for clinical neurologists, and the advent of such a promising therapeutic intervention rightfully deserves to be welcomed with enthusiasm. However, in the face of the documented, potentially harmful consequences of its overzealous administration, it may be wise to argue that it is those physicians who realize the caution and judgment which must be associated with the recent endorsement of t-PA who are most likely to make more effective use of it.

\section{REFERENCES}

1. MacDermott AB., Mayer ML, Westbrook GL, Smith SJ., and Barker JL. (1986). NMDAreceptor activation increases cytoplasmic calcium concentration in cultured spinal chord neurons. Nature, 321: 519-522.

2. Choi DW. (1995). Glutamate neurotoxicity and diseases of the nervous system. Neuron 1: 623-634.

3. Hardman and Limbird: Goodman and Gilman's The pharmacological basis of therapeutics, 10th ed., McGraw-Hill, 2001.

4. Cotran: Robbins Pathologic Basis of Disease, 6th ed., W. B Saunders Company, 1999.

5. Lindsberg PJ, Roine RO, Tatlisumak T, et al. The future of stroke management. Neurologic Clinics 2000;18:495-510.

6. Katzan IL, Sila CA, Furlan AJ. Community use of intravenous tissue plasminogen activator for acute stroke. Results of the Brain Matters Stroke Management Survey. Stroke 2001;2:861865

7. Fieschi C, Argentino C., Lenzi GL, Sacchetti ML, et al. (1989) Clinical and instrumental evaluation of patients with ischemic stroke within the first six hours. Journal of Neuroscience 91: 311-322.

8. The National Institute of Neurological Disorders and Stroke tPA Stroke Study Group (1995). Tissue plasminogen activator for acute ischemic stroke. New England Journal of Medicine, 333:1581-1587.

9. Kwiatkowski TG, Libman RB, Frankel M, Tilley BC, et al. (1999). Effects of tissue plasminogen activator for acute ischemic stroke at one year. New England Journal of Medicine 340:1781-1787.

10. Clark WM, Wissman S, Albers G, et al. Recombinant tissuetype plasminogen activator (alteplase) for ischemic stroke 3 to 5 hours after symptom onset. JAMA 1999;282:2019-2026.

11. Katzan IL, Furlan A.J, Lloyd LE, Frank JI, et al. (2000). Use of tissue-type plasminogen activator for acute ischemic stroke: The Cleveland Area Experience. Journal of the American Medical Association 283: 1151-1158.

12. Karbalai H, Demchuk AM, Hoyte KM, Klein GM, et al. (1999). Effectiveness of tissue-type plasminogen activator for ischemic stroke: consequences of protocol violation. Oral presentation at: 240th American Heart Association International Conference on Stroke and Cerebral Circulation; February 5, 1999; Nashville, Tennessee.

13. Tanne D, Demchuk A, Kasner SE, et al. (1999). A large multinational investigation to predict t-PA-related symptomatic ICH in patients with acute ischemic stroke. Stroke, 30: 248. 
14. Hacke W, Kaste M, Fieschi C, et al. Intravenous thrombolysis with recombinant tissue plasminogen activator for acute hemispheric stroke: the European Cooperative Acute Stroke Study (ECASS). JAMA 1995;274:1017-1025.

15. Hacke W, Kaste M, Fieschi C, et al. Randomized double-blind placebo-controlled trial of thrombolytic therapy with intravenous alteplase in acute ischemic stroke (ECASS II). Lancet 1998;352:1245-1251.

16. Demchuk AM, Morgenstern LB, Krieger DW, et al. Serum glucose level and diabetes predict tissue plasminogen activatorrelated intracerebral hemorrhage in acute ischemic stroke. Stroke 1999;30:34-39.
17. Clark WM, Wissman S, Albers GW, Jhamandas JH, et al. (1999). Recombinant tissue-type plasminogen activator (alteplase) for ischemic stroke 3 to 5 hours after symptom onset. Journal of the American Medical Association, 282: 2019-2026.

18. Barber PA, Zhang J, Demchuk AM, et al. Why are stroke patients excluded from t-PA therapy? An analysis of patient eligibility. Neurology 2001; 56:1015-1020.

19. Hacke W, Kaste M, Fieschi C, Toni D, et al. (1995). Intravenous thrombolysis with recombinant tissue plasminogen activator for acute hemispheric stroke. Journal of the American Medical Association, 274:1017-1025. 